$\begin{array}{ll}\text { AUthor(s) } & \text { M. J. Schmitt } \\ & \text { C. J. Elliott } \\ & \text { K. Lee } \\ & \text { E. D. McVey }\end{array}$

\section{IMIT.AIMFH}

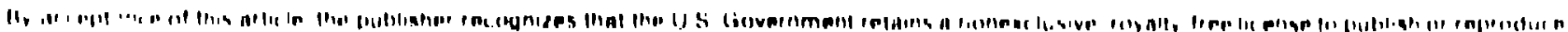

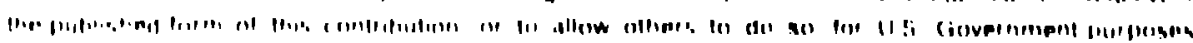

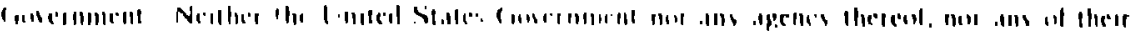

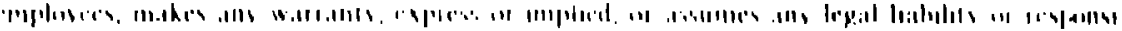

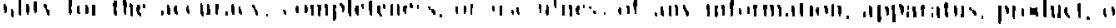

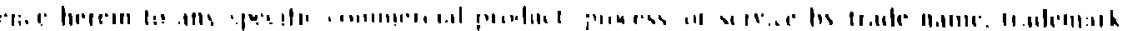

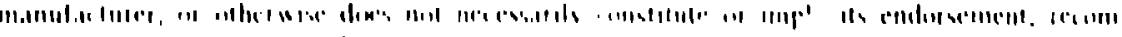

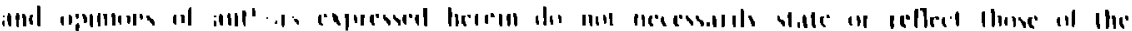

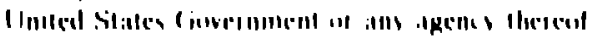




\title{
OVERTONE PRODUCTION OF SOFT X-RAYS WITH FREE-ELECTRON LASERS*
}

\author{
M. J. Schmitt, C. J. Elliott, K. Lee, B. D. McVny \\ Los Alamos National Laboratory, Los Alamos, NM \& 545
}

\begin{abstract}
Two one-dimensional free-electror laser codes have recently been written that include harmonic generation. A comparison of the results of these codes show that a self-consistent treatment of the harmonic interaction is not required in the presence of a strong fundamental field. Use of these codes to predict the effects of emittance on harmonic production have been conducted. The effects of wigg!er-field amplitude fluctuations and odd-harmonic wiggler-field components on the harmonic-radiation production are also discussed.
\end{abstract}

\section{INTRODUCTION}

The hat Inonics generated in free-electron laser (FFL) oscillator experiments are produced primarily through ce herent-spontaneous radiation of the fundamentally-bunched electron beam. T'his mechanism dominates over the much weaker harmonic linear-gain mechanism which cannot overcome cavity mirror losses. The amplitude of each harmonic is detcrmined by its fourier component of the transverse current, scaled by the harmonic coupling coeflicient.

This paper explores the effects of emittance, wiggler-ficld amolitude fluctuations and odd-harmonic wiggler-ficld components on harmonic production. A comparison between the one-dimensional codes is discussed in Section 1. In Section II the effects of emittance on the harmonic amplitudes are calculated assuming constant gain in the fundamental. Small amplitude fluctuations in the wighler magnetic field are modeled in Section III. Modifications of the harmonic coupling coefficients resulting from a small third-harmonic wiggler-fiold component are given in Section IV along with their consequences on harmonir production.

\section{HARMONIC: FHL (:OH)HS}

The equations describing the interaction of odd-harmonic frepueneiess with all electron beam in a Flil, are well documented", "These equations have been incorporated into a one-dimensional (1I)) code written by one of us (c.. J. lilliont)

* Work performed under the nuspices of, nnd supported by, the Division of Advaned binergy Projects of the U. S. Department of liner':y ()llice of Hasic Pincrgy Sciencers, 
to model both the radiation produced by the electron beam and the effect of the harmonic fields back on the electrons. This self-consistent code integrates the phase-averaged field equations given by

$$
\frac{\partial \varepsilon_{f}}{\partial x}=-2 \pi \rho K_{f}(\xi)\left\langle\frac{e^{-i\left(f \psi_{j}+\varphi_{0 f}\right)}}{\gamma_{j}}\right\rangle_{\text {electrone }}
$$

and the energy equation for each electron that states

$$
\frac{d \gamma_{j}}{d t}=\sum_{f=1}^{\infty} \frac{e \varepsilon_{f}}{2 m_{e} c \gamma_{j}} K_{f}(\xi) \cos \left(f \psi_{j}+\varphi_{0 j}\right)
$$

where $j$ denotes the $j$ th eleciron and $f$ is the harmonic number. This code has been compared to another code (HFELP) originaliy written by B. D. Mcley and later modified by $M$. J. Schmitt to include the harmonic radiation produced by a fundamentally bunched electron beam. The effects of the harmonic radiation fields on the electrons are not included in HFELP so that the electrons are only affected by the fundamental electromagnetic field that is assumed to be much larger than the harmonic fields.

To analyze the coherent-spontaneous emission of a FEL operating at the fundamental we assume the oscillator to have reached saturation so that a largo fundamental wave exists. A single-pass calculation is then performed assuming the faturated fundamental signal exists at the entrance to the wighler. This calculation is valid assuming the mirror losses at the harmonics prevent the harmonic radiation from circulating in the cavity. (iain at the fundamental is adjusied through an effective fill factor (') match that of the 3I) calculintion". The results of both codes, for input paramelers rescmbling these of the low Alamos osci!lator experiment ${ }^{4}$, are shown in Figures 1 a, b for L.e fifth harmonic.

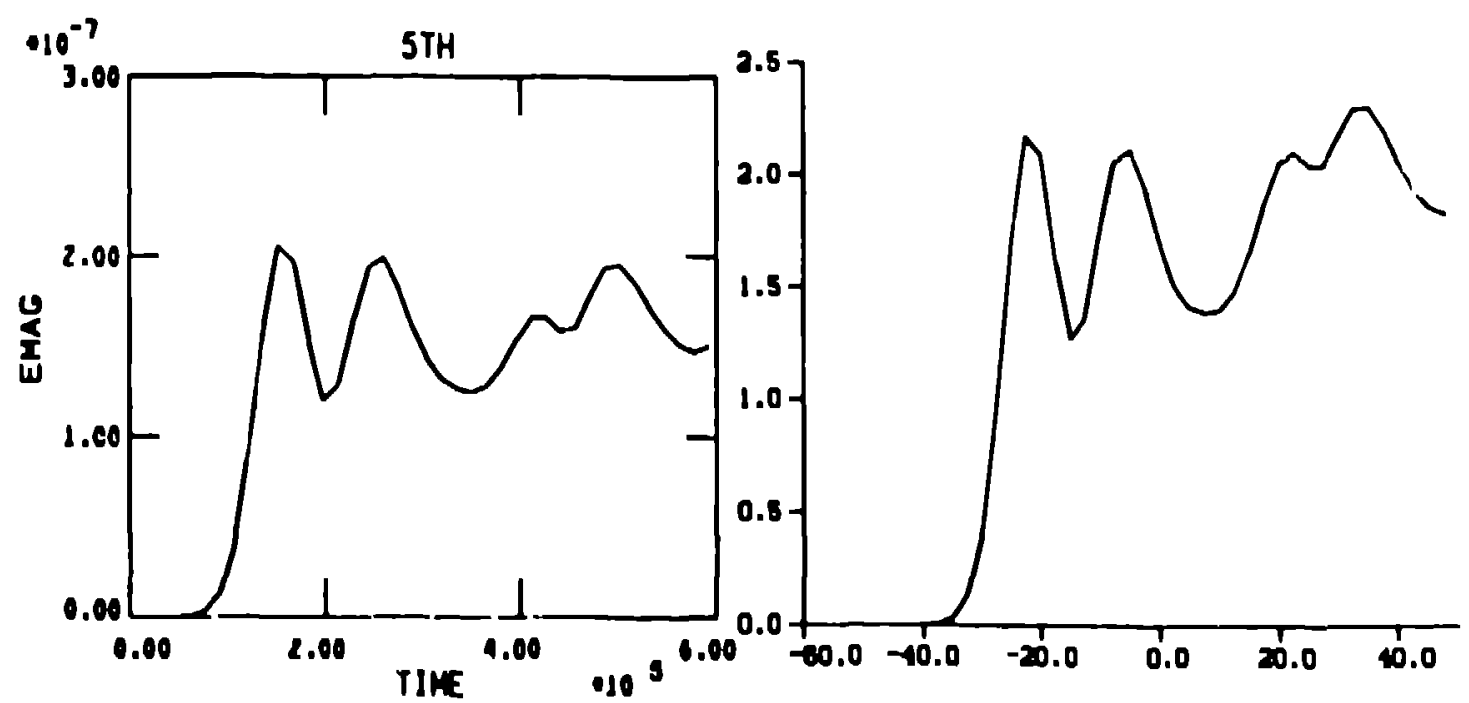

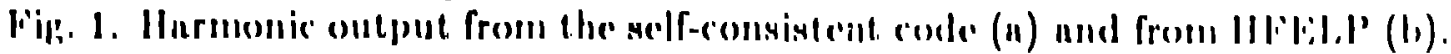


Results at the first few odd harmonics observed thus far all yicld identical results. From this comparison it is seen that for small tarmonic powers $\left(P_{f} / P_{1} \ll 1\right)$ a self-ronsistent analysis is noi required to obtain the correct harmonic output levels. Thus, calculation of the harmonic radiation can be done economically.

\section{EMITTANCE EFFECTS}

The growth of electron beam emittance has deleterious effects on FEL gain and efficiency. To evaluate how ernittance affects harmonic production, simulations were performed with the $1 D$ code HFELP. Owing to the one-dimensional nature of the code, electron-beam emittance was modeled as an effective electron energy spread. The energy spread represents the effective axial energy of the electrons. A typical distributicn for the electron beam used in the sinuiations for the proposed Los Alamos XUV FEI, ${ }^{5}$ is shown in Figure 2 for the case $c_{n}=40 \pi \mathrm{mm} \cdot \mathrm{mrad}$.

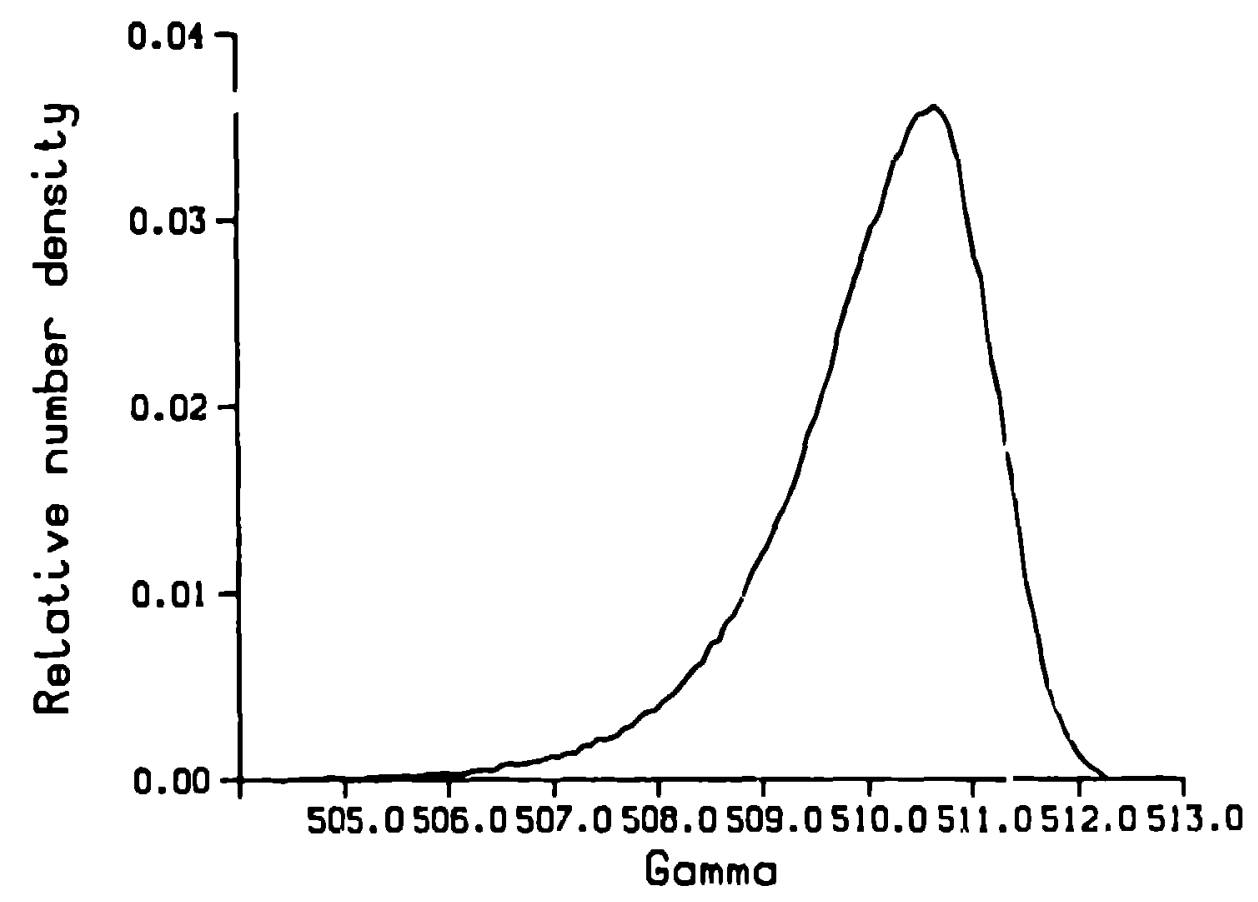

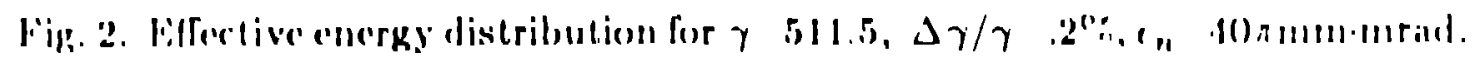

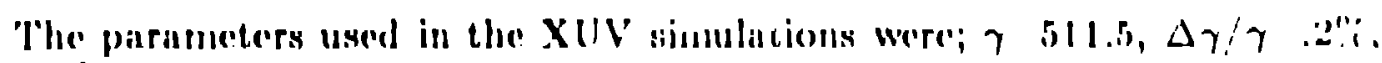

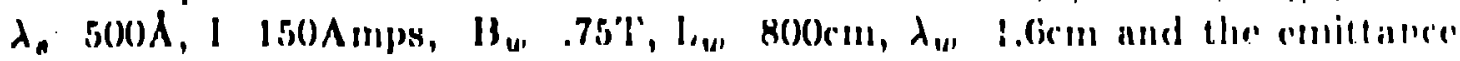
way varied from $20 \pi$ to $40 \pi$. The harmonic electric-field amplitudes at the end of the wiggler for these: conditions are plotted in ligure 3 for the first three odd-harmonirs. All harmonics show a significant reduction in ficld mmplitude. The third-harmonic amplitude shows the grealest eflect with a reduction of tent in field or two orders-of-magnilude :" power. 'Plhese results clearly show thial 


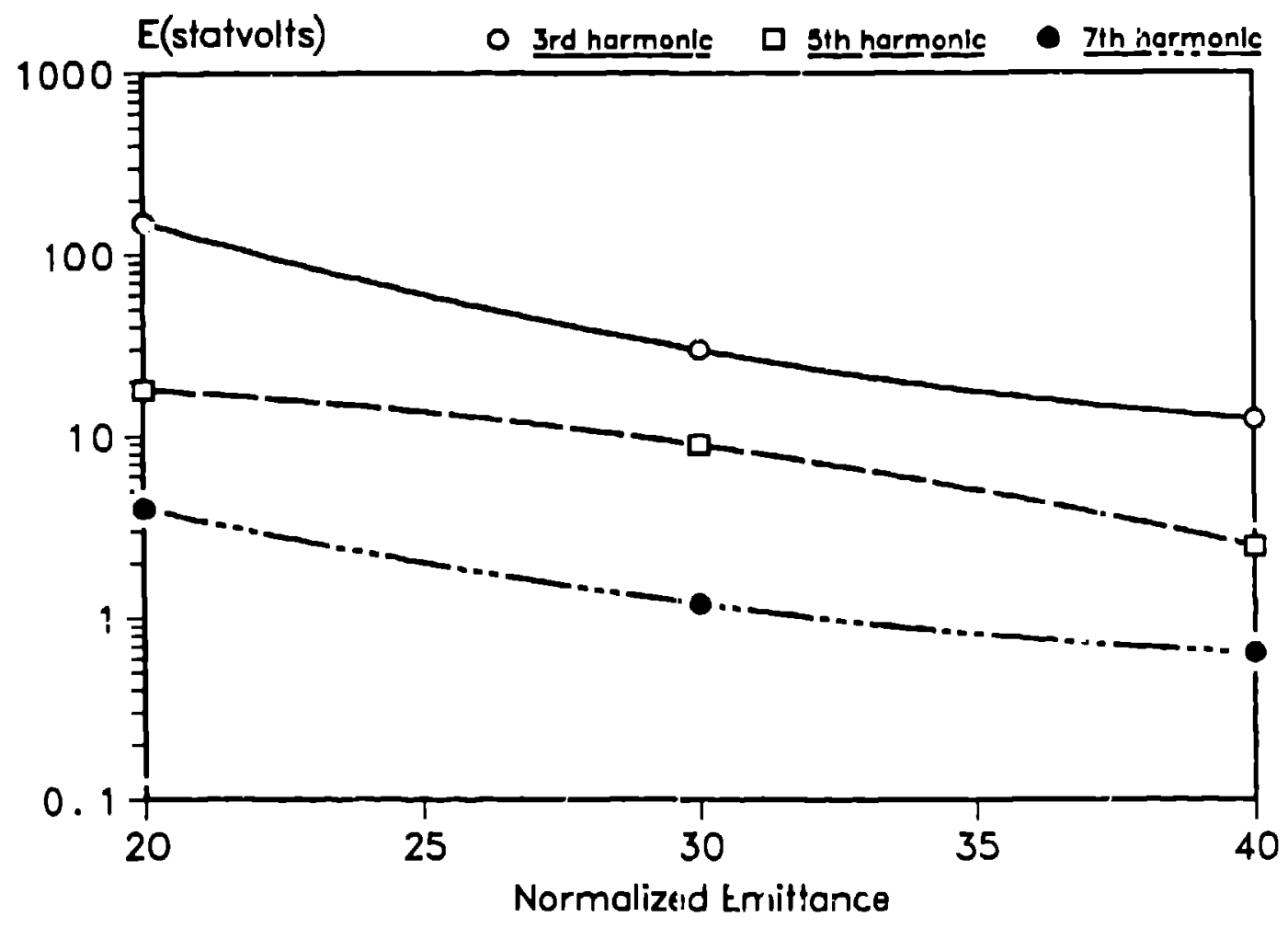

Fig. 3. ilarmonic electric-field amplitud.si as a function of normalized emittance

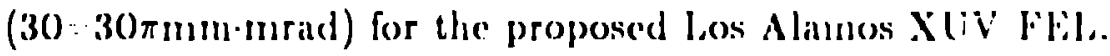

the minimization of emittance is critical for maximization of harmonic output.

\section{WIC(iLGR liliLl) ERRORS}

The magnetic field that results after the construction of a wiggler magnet is not a pure sinusoid. Differences in the magnetization of each magnet and machining tolepances guaranter that the resultant ficld will have varialions in both amplitude and wavelength. In this section $n$ : consider the effects of magnetis amplitude fluctuations on harmonic production. Although we are not treat-

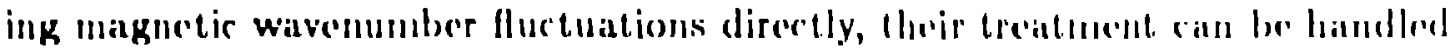
using this same formulism. Since this is a 11) treatment it is tacitly assumed that any random walk of the electron heam is compensated at the appropriate intervals to keep the electron and optical beimes concentric.

'Te model the variation of the amplitude of the wiggler field in the phasse. averaged equations one can calculate an effective variation in the clecelron phase $\checkmark$ due to the change in the magnetic-field amplitude. Ilecelectron phase in the wiggler and optical fields is given by

$$
\forall \quad\left(k_{1}, \mid k_{1}\right)=\omega t
$$

where st is determined by the average electron velocity in the axial direction. 
Assumir q a perturbation in the wiggler field amplitude given by $\Delta B_{w}$, a resultant perturbation in the axial position will result that will in turn produce a vaiation in the electron phase given by

$$
\Delta \psi=\left(k_{w}+k_{s}\right) \Delta \bar{z}
$$

and upon substitution for the appropriate value of $\Delta \bar{z}$ gives

$$
\Delta \psi_{j}=-\frac{\pi}{2} \frac{\left(1+k_{s} / k_{w}\right)}{\gamma_{j}^{2}} a_{w} \Delta a_{w}
$$

where $a_{w}$ is the dimensionless magnetic vector potential defined

$$
a_{w}=\frac{e B_{w}}{m_{e} c^{2} k_{w}} .
$$

Implementation of $\mathrm{Eq}(5)$ in the $1 \mathrm{D}$ code HFELP was conducted by calculating the phase error correction for each electron caused by small magnetic-field amplitude fluctuation at each wiggler half-period section. This correction was then added to the clectron's phase after it had traverse each wiggler half-period. The errors were assumed to lave a gaussian distribution with the $1 / e^{2}$ point defined as an input variable. Simulations were performed for the paraneters given in Section I! for the proposed Los Alamos XUV FEL assuming a $1 \%$ error in tixe magnetic field amplitude and an emittance of $20 \pi$. The results showed a 10 to $20 \%$ decrease in the electric field amplitude of the first few odd-harmonics concurrent with a $7 \%$ drcp in the fundamental electric-field amplitude.

Errors in wavenumber can likewise be treated b: calculating an effective phase change $\Delta \psi_{\lambda}=\Delta k_{u}, z$ and incorporating it into each electron's phase after every wiggler wayelength. A simple calculation shows that for the above Darameters a $1 \%$ wiggler magnetic-field amplit.de fluctuation is equivalent to a $.75 \%$ fluctuation in the wiggler wavelength. Thus, wiggler amplitude and wavenumber errors should be kept below $1 \%$ for harmonic amplitudes to remain unelfected.

\section{IARMONIC; WIG(BLER FIELI) COMPONEN'IS}

'To increase the stecngth of the fibl interastion, experunenters strive to acheve normalized magnetic vector potentials of order unity or greater. 'The construction of such wigglers results in highly peiked magnetic ficld intensities neitr the pole facess significantly deviating from the analytically assumed sinusoid. A fourier deromposition of these fields yicld third-harmonic lichd compornents approaching $20 \%$ or more that of the fundamental". The effects of these harmonic wiggler fields cannot be includeci by linear superposition. We have calculated how these components change the coupling cocflicientes bor the oddharmonic radiat ion and examine below how a small third-harmonic wiggler lield modifies the fundamental and third-harmonic rndintion. 
To include the effect of a third-harmonic wiggler field we first define the t:ansverse velocity produced by a sum of harmonic wiggler ficlds as

$$
\beta_{\perp}^{2}=\left(\frac{e A_{w}}{m_{e} c^{2} \gamma}\right)^{2}=\frac{e^{2}}{m_{e}^{2} c^{4} \gamma^{2}}\left\{\sum_{m=1}^{\infty} A_{w m} \cos \left(m k_{0} z\right)\right\}^{2}
$$

which can be used to derive a new resonance cordition in the high- $\gamma$ limit given by

$$
k_{0}=\frac{k_{a}}{2 \gamma_{0}^{2}}\left\{1+\sum_{m=1}^{\infty} \frac{a_{w}^{2} m_{2}}{2}\right\}
$$

These equations can then be used to derive new coupling cocflicients for use in Eqs(1),(2). Assuming only a third-harmunic wiggler field with $a_{w 3} / a_{u, 1} \ll 1$, the new coupling terms for the fundanental and third-harmonic are

$$
\begin{aligned}
\mathcal{K}_{1}(\xi)= & J_{0}\left(\xi_{1} \frac{a_{w 3}-\frac{1}{a_{u 1}}}{a^{\prime}}\right) J_{v}\left(\xi_{3}\right) \\
& \cdot \mid a_{u^{\prime} 1}\left\{J_{0}\left(\alpha_{1}\right)-J_{1}\left(\alpha_{1}\right)\right\}+a_{w 3}\left\{J_{1}\left(\alpha_{1}\right)+J_{2}\left(\alpha_{1}\right)\right\} !
\end{aligned}
$$

and

$$
\begin{aligned}
\mathcal{K}_{3}(\xi)- & J_{0}\left(3 \xi_{1} \frac{a_{u 3}}{a_{u 1}}\right) J_{0}\left(3 \xi_{31}\right) \\
& \cdot\left|a_{u 11}\left\{J_{2}\left(\alpha_{33}\right)-J_{1}\left(\alpha_{3}\right)\right\}+a_{w 3}\left\{J_{0}\left(\alpha_{3}\right)-J_{33}\left(\alpha_{3}\right)\right\}\right|
\end{aligned}
$$

where

$$
n \cdot n \xi_{1}\left(1,2^{a} \begin{array}{l}
a_{u}: 3 \\
a_{u}+1
\end{array}\right)
$$

and

$$
\xi_{m} \cdot a_{u m}^{2}\left(1+\sum_{n} \sum_{1,3} a_{u n}^{2}\right)^{\prime}
$$

A compnrisen of these new coefliciont: (assuming a $10 \%$ thidd-harmonic contribution) with the old ${ }^{1}$ coefficionts for paratmeters consistent with those of the los Alamos oscillator experiment show that while the fundiament al term is unaffected, the this d-harmonic term hat changed by 33 "'o. Note that we have atswumed the magnitude of $a_{w 3}$ opposite that of $a_{w 1}$ such that the resultant magnetic ficld is more praked than a sinusoid. For this configuration the third-harmonic power is conhanced by $75 \%$. This; example dramatically shows how the harmonics can be affected be harmonic wiggler components. A more thorough analysis of this effect is presented clsewhere?. 


\section{REFERENCES}

1. W.E. Colson, IEEE JQE QE-17, 1417 (1981).

2. J.M.J. Madey and R.C. Taber, Physics of Quantum Electronics, vol. 7, Jacobs et al.,Eds., (Addison-Wesley, Keading, MA, 1980), ch. 30.

3. B.D. McVey, in Proceedings of the Seventh International Conference on Free-Electron Lasers, held in Tahoe City, CA, 1985, in publication.

4. B.E. Newnam et al., IEEE JQE QE-21, 867 (1985).

5. J.C. Goldstein et al., in Proceedings of the Internoliunal Conference on Insertion Devices for Synchrotron Sources. held in Sianford, CA, on Oct. 27-30, 1985, to be published.

6. K. Haibach, Journal de Physic,ue, Ćrıloque $C 1$, supplément au $n^{\circ} 2$, Tome 44, février 1983.

7. M.J. Schmitt and C.J. Elliott, to be published. 